\title{
Observations on the Therapeutic Effect of Different Frequency Electroacupuncture on Poststroke Hemidysesthesia
}

\author{
Teng Xiuying ${ }^{1, *}$, Gao Changle ${ }^{1,2}$, Wang Chenran², Du Xiaowei ${ }^{2}$, Zhang Ruyue $^{2}$, He Xu$^{2}$ \\ ${ }^{1}$ The Second Affiliated Hospital of Heilongjiang University of Traditional Chinese Medicine, Harbin, P. R. China \\ ${ }^{2}$ Rehabilitation Medicine of Traditional Chinese Medicine, Heilongiiang University of Traditional Chinese Medicine, Harbin, P. R. China \\ Email address: \\ 2281961458@qq.com (Gao Changle), xiuyingteng2006@126.com (Teng Xiuying) \\ ${ }^{*}$ Corresponding author
}

To cite this article:

Teng Xiuying, Gao Changle, Wang Chenran, Du Xiaowei, Zhang Ruyue, He Xu. Observations on the Therapeutic Effect of Different Frequency Electroacupuncture on Poststroke Hemidysesthesia. International Journal of Chinese Medicine. Vol. 3, No. 2, 2019, pp. 25-29. doi: $10.11648 /$ j.ijcm. 20190302.13

Received: May 5, 2019; Accepted: June 13, 2019; Published: July 1, 2019

\begin{abstract}
Objective to observethe clinical effect of electroacupuncture with different frequency in the treatment of hemidysesthesia after stroke. Methods Sixty patients with hemidysesthesia after stroke were randomly divided into treatment group and control groups, with 30 cases in each group. Both groups were treated with electroacupuncture with the same acupoint selection. The electroacupuncture frequency was $100 \mathrm{~Hz}$ in the treatment group and $1 \mathrm{~Hz}$ in the control group. The clinical effficacy of the two groups was observed after 30 days of treatment. Results The total effective rate and the recovery rate of the treatment group were $93.3 \%$ and $80.0 \%$, respectively, and the control group were $86.0 \%$ and $43.3 \%$, respectively The difference in the total effective rate between the two groups was statistically significant $(p<0.05)$. And the difference in the recovery rate between the two groups was statistically significant $(p<0.05)$. There were statistically significant differences in the scores of limb dysesthesia score between the two groups after treatment and before treatment $(p<0.01)$. There was a statistically significant difference in the limb dysesthesia score after treatment between the treatment group and control group $(p<0.05)$. Conclusions Electroacupuncture is an effective method to treat poststroke hemidysesthesia. High frequency electroacupuncture is more obvious than low frequency electroacupuncture.
\end{abstract}

Keywords: Acupuncture Therapy, Dysesthesia Hemidysesthesia, Electroacupuncture, Scalp Acupuncture, Electroacupuncture Frequency

\section{Introduction}

Hemidysesthesia is a common symptom after stroke, and it is also the difficulty of treatment. It has been reported that about $65 \%$ of stroke patients have different degrees and types of sensory disorders, among which hemisensory disorders account for about 50\% [1-2]. (The symptoms of sensory disturbance can be divided into two categories. On the one hand is isIrritant symptoms, which include pain, hyperesthesia, paresthesia, severe dysesthesia, and paresthesia compared to normal. On the other hand is inhibitory symptoms, which include anaesthesia or hypoesthesia.) (Dysesthesia has a significant impact on physical movement, coordination, balance etc. [3], which seriously affects patients' quality of life and rehabilitation. Besides, dysesthesia would also lead to a lot of pain to patients.)

Our study found that acupuncture and moxibustion treatment of dysesthesia after stroke has clear curative effect, and electric acupuncture has simple operation, saving manpower, strengthen the acupuncture therapy effect, safe use, etc, and it has been widely used at home and abroad among doctors, however, in recent years the study of treatment of sensory disturbance method for acupuncture and meridians to explore more, for the treatment of dysesthesia of the selection of optimal frequency is unclear. Therefore, this study intends to further observe the effect of different frequency electroacupuncture stimulation on its clinical treatment effect, which is reported as follows. 


\section{Clinical Data}

\subsection{General Information}

Sixty patients with hemiparesthesia after stroke were all patients in the second ward or outpatient department of the rehabilitation department of the second affiliated hospital of Heilongjiang Uiversity of Traditional Chinese Medicine from May 2017 to May 2018. According to the order of treatment, the patients were randomly divided into treatment group and control group with 30 patients in each group. In the treatment group, there were 17 males and 13 females. The youngest was 42 years old, and the oldest was 70 years old, with an average $(58 \pm 8)$ years old. The course of the disease was the shortest 1 $\mathrm{d}$ and the longest $14 \mathrm{~d}$, with an average $(9.56 \pm 4.72) \mathrm{d}$. In the control group, there were 16 males and 14 females. The youngest was 44 years old, and the oldest was 69 years old, with an average $(58 \pm 7)$ years old. The duration of the disease was the shortest $(1 \mathrm{~d})$ and the longest $(14 \mathrm{~d})$, with an average $(9.84 \pm 4.65) \mathrm{d}$. There was no significant difference in gender, age and disease course between the two groups $(>0.05)$, which was comparable.

\subsection{Diagnostic Criteria}

\subsubsection{Diagnostic Criteria of Western Medicine}

The diagnosis of various cerebrovascular diseases was revised by the 4th national conference on cerebrovascular diseases of the Chinese medical association in 1995 [4].

\subsubsection{TCM Diagnostic Criteria}

The criteria for evaluating the diagnostic efficacy of apoplexy (trial) drafted by the cerebrovascular emergency cooperation group of the state administration of traditional Chinese medicine was adopted [5].

\subsection{Inclusion Criteria}

(1) In line with the traditional Chinese medicine diagnosis of stroke and western medicine diagnosis of cerebrovascular disease patients; (2) Diagnosed as ischemic cerebral infarction or cerebral hemorrhage by head CT or MRI; (3) The age is 40 70 years old; (4) Accompanied by different degrees of limb dysesthesia (before the onset of the disease without limb dysesthesia), the body sensory evaluation score 35 points; (5) The patient has clear consciousness and normal spirit, and has the ability to express on the examination; (6) Patients can accept and cooperate with acupuncture treatment, the course of the disease within 14 days; (7) Signed a consent form.

\section{Treatment}

Pick scalp acupuncture sensory and patients suffering from points on the side, the upper limbs take Jianyu (LI15), Quchi (LI11), Shousanli (LI10), Waiguan (TE5), Hegu (LI4); the lower limbs takeBiguan (ST31), Xuehai (SP10), Sanyinjiao (SP6), Xuanzhong (GB39).[6] Use 0.30mm (diameter) $\times 40 \mathrm{~mm}$ (length) acupuncture needle after routine disinfection. After needle insertion obtining qi, take Jianyu (anode) and
Quchi (cathode), Shousanli (anode) and Waiguan (cathode), Biguan (anode) and Xuehai (cathode), Yanglingquan (GB34) (anode) and Xuanzhong (cathode) connect with KWD-808 type I pulse acupuncture therapeutic apparatus, adopt continuous wave, frequency of electric acupuncture treatment group of $100 \mathrm{~Hz}$, control group $1 \mathrm{~Hz}$, retaining needle for 30 min. Both groups received once a day for 30 consecutive days.

\section{Therapeutic Effect}

\subsection{Observation Indicators}

\subsubsection{Score of Limb Dysesthesia}

Before and after treatment, the improved fugl-meyer and Lindmark evaluation [7-8] were used to record the score of limb sensory impairment in two groups. The highest score is 42 points, and the lowest score is 0 points (unilateral limb), among which the highest score is 20 points for shallow sensation (touch, pain and temperature sensation) and the lowest score is 0 points. Cortical sense (two-point discrimination sense, solid sense) the highest score is 6 points, the lowest score is 0 points; The highest score of proprioception (motion sense, position sense) is 16 points, and the lowest score is 0 points.

\subsubsection{Classification of Dysesthesia Degree}

The degree of patient self-reported limb dysesthesia (including superficial sensation, cortical sensation and proprioception) was the auxiliary index. The scores were $0 \sim$ 15 for severe, $16 \sim 25$ for moderate and $26 \sim 35$ for mild. Patients who scored $>35$ points were not included in the scope of observation, in order to avoid the integral error caused by subjective and objective reasons and to influence the objectivity of clinical observation.

\subsubsection{Attention}

In the examination and evaluation of patients should pay attention to patients with normal consciousness and spirit, to ensure that patients can cooperate with the examination and express clearly; Patients should be closed eyes examination in order to prevent the suggestion (In order to avoid errors caused by fatigue, the time of examination should be well controlled, and the best time should not exceed $15 \mathrm{~min}$.) (If you are not sure once, do it again.) Paying attention to the objective conditions and comprehensive analysis according to the subjective reaction.

\subsection{Curative Effect of Standard}

Recovery: the total score after treatment is 25 points higher than before treatment, and the total score after treatment is 40 points; If it is a single dysesthesia, the maximum score should reach above $90 \%$ (including $90 \%$ ) after treatment. At the same time, the patients' self-reported symptoms has disappeared, and there was no significant difference with healthy limbs.

Significant effect: the score after treatment was 15 points higher than before treatment, but the total score was $<40$ points; if it is a single dysesthesia, the maximum score should be over $70 \%$ (including $70 \%$ ) after treatment. At the same 
time, the self-reported symptoms were significantly better than before treatment.

Effective: the total score after treatment is 5 points higher than before treatment; If it is a single dysesthesia, the maximum score should be reach 50\% (including 50\%) after treatment. At the same time, the patients' self-reported symptoms were alleviate than before treatment.

Inefficacy: the total score after treatment was less than 5 points higher than before treatment. For single dysesthesia, the score after treatment is less than $50 \%$ of the maximum score; At the same time, the patients' self-reported symptoms were not significantly changed compared with those before treatment.

In addition, when the score is inconsistent with the degree of self-reported symptoms, the degree of improvement in order to the patients' self-reported symptoms shall prevail.

\subsection{Statistical Methods}

All data were statistically analyzed using SPSS 17.0 software. The measurement data were expressed as mean standard deviation and tested. The chi-square test was used for counting data. Rank sum test was used for rank data. The difference was statistically significant if $(p<0.05)$.

\subsection{Treatment Results}

Comparison of Clinical Efficacy Between the Two Groups

As can be seen from Table 1, the total efficacy rate and the cure and marked efficacy rate were $93.3 \%$ and $80.0 \%$, respectively, in the treatment group and $86.0 \%$ and $43.3 \%$, there was a statistically significant difference in the cure and marked efficacy rate between the two groups $(p<0.05)$.

Table 1. Comparison of clinical efficacy between the two groups.

\begin{tabular}{llllllll}
\hline Group & Number of people & Recovery & Significant effect & Effective & Inefficacy & Marked effective rate/\% & Total effective rate/\% \\
\hline TG & 30 & 9 & 15 & 4 & 2 & $80.0^{1)}$ \\
CG & 30 & 4 & 9 & 13 & 4 & 93.3 \\
\hline
\end{tabular}

Comparison with control group ${ }^{1)} P<0.05 ; \mathrm{TG}=$ Treatment group; $\mathrm{CG}=$ Control group.

\subsection{Comparison of Scores of Limb Dysesthesia Before and After Treatment Between the Two Groups}

As can be seen from Table 2, there was no statistically significant difference between the two groups in the score of limb dysesthesia before treatment $(p>0.05)$. After treatment, the scores of limb dysesthesia in the two groups were compared with those before treatment in the same group, and the differences were statistically significant $(p<0.01)$. There was a statistically significant post-treatment difference in the limb dysesthesia score between the treatment and control groups $(p<0.05)$.

Table 2. Comparison of scores of limb dysesthesia before and after treatment between the two groups ( $x \pm s$, score).

\begin{tabular}{llll}
\hline Group & Number of people & Before treatment & After treatment \\
\hline TG & 30 & $15.32 \pm 5.17$ & $29.53 \pm 5.34^{12)}$ \\
CG & 30 & $15.74 \pm 4.73$ & $25.71 \pm 6.23^{1)}$ \\
\hline
\end{tabular}

Compared with the same group before treatment ${ }^{1)} P<0.01$; Comparison with control group ${ }^{2)} P<0.05 ; \mathrm{TG}=$ Treatment group; $\mathrm{CG}=$ Control group.

\section{Discussion}

Traditional Chinese Medicine thought that, poststroke hemiparesthesia is due to the body, and resulted in the adverse operation of defense qi, nutrient and blood, external pathogen encumber meridians and collaterals. For example, in LingShu.CiJieZhenXie: "genuine qi cannot fill the entire body, pathogen had invaded upper and lower limbs of one sided, into the deep within the nutrient and defence, when qi dynamic disorder, genuine qi leave, and only pathogen leave behind, then hemiplegia."Su Wen·Ni Diao Lun: "nutrient qi deficiency give rise to numbness, that means insensitivity. Defense qi deficiency is not used, dual deficiency of nutrient and defense are numbness and futility. "Su WenFFeng Lun: "defense qi congeal and rough that cannot run, numbness of the skin, cannot felt pain and itch." Huang Yuanyu in the qing dynasty, the four sacred heart sources: stroke roots:" Yang deficiency and soil wet, middle qi can not reach all parts of body, limbs meridians and collaterals, congeal and wet but not transport, defense qi obstruct, so numbness. "Therefore, the treatment principle is to harmonize the nutrient and defense and dredge the meridians and collaterals."Huang Di Nei Jing:"want to use filiform needle, free the meridian vessels, harmonize qi and blood." This provides a theoretical basis for acupuncture treatment. Compared with traditional acupuncture and moxibustion, electroacupuncture can save manpower, Which avoid the disadvantage of small amount of traditional acupuncture stimulation, enhance the efficacy and promote the recovery of nerve function [9]. This study method is to use traditional acupuncture technique with pulse current constant stimulation, the scalp sensory area cooperates with side local take point, electroacupuncture scalp area can cause body feeling cortex and brain cortex excitability change reaction [10-11], expand blood vessel, increase cerebral blood flow, establishing collateral loop [12], improve cerebral ischemia oxygen deficit, promote central nervous function restore; Local acupoint selection for meridian circulation on the affected side and electroacupuncture stimulation can accelerate local blood circulation, free the meridian and collateral vessels, qi and blood, and harmonize the nutrient and defense without attachment of pathogenic factors such as wind, cold, dampness and heat, so as to treat on poststroke hemidysesthesia.

Relevant studies have shown that electroacupuncture of different frequencies can induce cerebral ischemia tolerance to varying degrees [13], but its effects are different. Low frequency electroacupuncture promoted PPE expression [14]; High-frequency electroacupuncture promoted the expression 
of endogenous neurotrophic factors GDNF and BDNF and inhibited the activation of microglia cells [15-16]. From the perspective of cell electrophysiology, high-frequency electroacupuncture stimulation also has the effect of "preemptive occupation", which can inhibit pathological nerve impulses and conduction [17], reduce abnormal nerve impulses, and thus reduce poststroke hemidysesthesia [18-21]. From the perspective of rehabilitation medicine, poststroke dysesthesia are mainly treated through occupational therapy to enhance a variety of sensory inputs, so that patients gradually improve the ability to feel pain [22-25], while high-frequency electroacupuncture can more stimulate deep pain receptors and skin pain receptors [26-28]. Wu Zhengji found that electroacupuncture with different frequency rates could improve neural function defects in rats, but electroacupuncture with high frequency had more advantages in improving nerve growth factor protein in peripheral brain tissue than electroacupuncture with low frequency. [29] Ye Xiaochun ect. found that electroacupuncture stimulation of different frequencies has certain improvement effect on pain or numbness, among which the effect of high-frequency electroacupuncture is the most obvious. [30]

This study result shows that the electricity of poststroke hemidysesthesia has obvious therapeutic effect, in which high frequency electric acupuncture therapy than low frequency electric acupuncture therapy effect is more apparent $(<0.05)$, poststroke hemidysesthesia has better reflect to the high frequency electric acupuncture, for clinical use electroacupuncture help poststroke hemidysesthesia to a certain extent, but the test take a small sample of clinical observation, study time and the number of cases is limited, is a preliminary discussion for the electroacupuncture treatment of poststroke hemidysesthesia. Hope to have a large sample of clinical observation and animal experiment, provide more reliable basis for treatment of these diseases and train of thought.

\section{Conclusion}

The results of the study demonstrated that acupuncture has a good promoting effect on the treatment of hemidysesthesia after stroke, with few adverse reactions and good tolerance. According to the data statistics, the changes of high frequency electroacupuncture are more meaningful, which also proves that electroacupuncture therapy is safe and reliable.

\section{References}

[1] Wang YY, Research situation and development trend of Traditional Chinese Medicine [J]. Chinese Journal of basic medicine of Traditional Chinese Medicine, 2011, 17 (7): 705-707.

[2] Wei XP, Yao BN, Zheng J. Clinical observation on 30 cases of stroke dysesthesia treated by Duhuojishengtang combined with Zhuang medicine line point moxibustion [J]. Hunan Journal Traditional Chinese Medicine, 2013, 29 (6): 61-63.
[3] Tan YX, Qi XK. Effects of limb motor function rehabilitation on limb sensory recovery in stroke patients with hemiplegia [J]. Chinese Journal of Neurology, 2009, 42 (8): 538-539.

[4] Chinese Neurology Society, Chinese Society of Neurosurgery, Diagnostic essentials of various cerebrovascular diseases [J], Chinese Journal of Neurology, 1996, 29 (6): 379-380.

[5] Cerebrovascular emergency cooperation group, state administration of Traditional Chinese Medicine. Evaluation criteria for stroke diagnosis and therapeutic effect (trial) [J]. Journal of Beijing university of Chinese medicine, 1996, 19 (1): 55-56.

[6] Gao WB. Acupuncture and moxibustion in six parts modern acupuncture and moxibustion therapy for neuropathy $[\mathrm{M}]$. Beijing: China Medical Science and Technology Publishing Huose, 2007: 136.

[7] Sun G, Zhou ZL, Mao L, et al. etc. Treatment of poststroke hemidysesthesia with wrist-ankle acupuncture [J]. Chinese Acupuncture, 2011, 31 (1): 35-39.

[8] Zhou ZL, Yang JR. Observation on curative effect of needle blood therapy on hemidysesthesia of cerebrovascular diseases [J]. Shanghai Journal of Acupuncture and Moxibustion, 2004, 23 (5): 6-7.

[9] Gong GQ, Bilateral electroacupuncture for treatment of stroke sequelae in 40 cases [J]. Journal of Traditional Chinese Medicine, 2006, 47 (6): 448.

[10] $\mathrm{Wu} \mathrm{H}, \mathrm{Gu} \mathrm{XD}, \mathrm{Wu} \mathrm{CH}$, et al. etc. Clinical study of electroacupuncture stimulation of scalp sensory area on functional recovery of patients with stroke [J]. Chinese Journal of Traditional Chinese Medicine, 2013, 28 (8): 2463-2466.

[11] $\mathrm{Hu} \mathrm{H}$, Wang GX, Han YS, et al. etc. Clinical study on electroacupuncture treatment of stroke in head and napex [J]. Journal of Clinical Chinese Medicine, 2015, 27 (7): 913-917.

[12] Li GP, Shi L, Du YH, et al. Study on collateral circulation reconstruction and electroacupuncture intervention effect in early stroke $[\mathrm{J}]$. Journal of Tianjin University of Chinese Medicine, 2011, 30 (2): 102-104.

[13] Lao N, Xiong LZ, Lu ZH, et al. etc. Difference in tolerance of different frequency electroacupuncture pretreatment to induced cerebral ischemia $[\mathrm{J}]$. Chinese Journal of Clinical Rehabilitation, 2005, 9 (9): 116-117.

[14] Wang YW, Chen HD. Different frequency electroacupuncture effect of experimental and clinical studies. Journal of Anhui University of Traditional Chinese Medicine, 2006, 25 (3): 58-61.

[15] Liang XB, Luo Y, Liu XY, et al. etc. Electroacupuncture3. Improves behavior and upregulates GDNF mRNA in MFB transected rats [J]. Neuroreport, 2003, 14 (8): 1177-1181.

[16] Liu XY, Zhou HF, Pan YL, et al. etc. Electroacupuncture Stimulation protects dopaminergic dogs [J]. Exp Neurol, 2004, 189 (1): 189- 196.

[17] Guo JH, Zhang BN, Zhu ZY, et al. etc. Electroacupuncture with different frequency for the treatment of Trigeminal neuralgia. [J]. Chinese Acupuncture and Moxibustion, 2006, 26 (12): 903.

[18] Wang RY, Lin XL, Sun QH. Clinical study on intervention of poststroke fatigue by percutaneous electrical stimulation of points [J]. Shanghai Journal of Acupuncture and Moxibustion, 2017, 36 (1): 14-16. 
[19] Zhang LF, Wang LY, Li LY, et al. etc. Effect of the scalp electroacupuncture combined with mandatory exercise on upper limb function in patients with ischemic stroke [J]. Chinese Journal of Traditional Chinese and Western Medicine, 2017, 37 (3): 314-318.

[20] Yao SQ. Effect of electroacupuncture on point JiQuan on upper limb function in stroke patients [J]. Gansu Science \& Technology, 2015, 31 (9): 132-133.

[21] Niu B. Comparison of curative effect of electroacupuncture and neuromuscular electric stimulation therapy in treatment of swallowing disorders caused on stroke [J]. Massage and Rehabilitation Medicine, 2015, 6 (24): 40-41.

[22] Chen YL, Zhou KB. Clinical efficacy analysis of Traditional Chinese Medicine combined with acupoint injection in the treatment of hemiparesthesia after stroke [J]. China Medical Journal, 2012, 9 (25): 123-124.

[23] Zhou MM, Liu FY, Yue LP, et al. etc. Electroacupuncture changes inflammation and pain in CFA rats electro-discharge activity of neurons in the anterior cingulate gyrus [J]. Chinese Journal of Pain Medicine, 2016, 22 (10): 737-742.

[24] Qin GH, Ma Y, Zhao X, et al. etc. Behavior observation of different frequency electroacupuncture stimulation to relieve pain emotion in rats [J]. Chinese Medical Innovation, 2017, 14 (14): 1-4.
[25] He XF, Fang JQ, Jiang YL, et al. etc. Electroacupuncture promote beta-END peripheral release for the treatment of chronic inflammatory pain. Shanghai Journal of Acupuncture and Moxibustion, 2013, 32 (5): 407-409.

[26] Dong QS, Zhang RT, Dong XM, et al. etc. Probe into acupoint material basis of electroacupuncture sensation from the relationship between electroacupuncture sensation and sensory function [J]. Sichuan Traditional Chinese Medicine, 2007, 25 (6): $95-100$

[27] Huang C, Wang Y. Discussion on the mechanism of low-frequency and high-frequency electroacupuncture analgesic opioid in mice $[\mathrm{J}]$. Chinese Journal of Pain Medicine, 2000, 6 (2): 96-103.

[28] Chen SX, Wang YW. Effect of high-frequency electroacupuncture combined with thermal moxibustion in the treatment of 35 cases of knee osteoarthritis [J]. Zhejiang Journal of Traditional Chinese Medicine, 2013, 48 (11): 833.

[29] $\mathrm{Wu} \mathrm{ZJ}$. Effects of different frequency electroacupuncture on nerve growth factor and vascular endothelial growth factor in rats with cerebral hemorrhage [D]. Guangzhou University of Chinese Medicine, 2008.

[30] Ye XC, Ba DJ, Zhao P, et al. etc. Clinical study on different frequency electroacupuncture stimulation in the treatment of cervical spondylotic radiculopathy [J]. Shanghai Journal of Traditional Chinese Medicine, 2015, 49 (4): 65-67. 